\title{
Comparative Study on Various Numerical Schemes for Solving Nozzle Flow Problems
}

\author{
Nurhayati Rosly ${ }^{1, *}$, Qamarul Ezani Kamarudin², Bambang Basuno ${ }^{1}$ \\ 1 Department of Aeronautical Engineering, Faculty of Mechanical and Manufacturing Engineering, Universiti Tun Hussein Onn Malaysia, 86400 \\ Parit Raja, Johor, Malaysia \\ 2 Aircraft System and Design Research, Faculty of Mechanical and Manufacturing Engineering, Universiti Tun Hussein Onn Malaysia, 86400 Parit \\ Raja, Johor, Malaysia
}

\section{ARTICLE INFO}

\section{Article history:}

Received 21 April 2020

Received in revised form 18 June 2020

Accepted 25 June 2020

Available online 30 June 2020

\section{ABSTRACT}

The inviscid compressible flows represent the flow model of high-speed flow with the viscous effects can be ignored. Such flow phenomena appear when a high Reynolds number flow past trough a streamlined body at relative a low angle attack. The most commercial passenger airplane is operated at such flow condition, as results the aircraft industries have already put their efforts to develop the capability for solving the governing equation of inviscid compressible flow. The governing equation of inviscid compressible flow which is called as the Euler Equation can solved numerically. There are various numerical schemes had been developed and the most of them are dedicated for capturing the presence of shock wave phenomena which is normally appearing at high speed flow. The present work presents a comparative study over various numerical schemes which they are already been developed for solving the Euler equations. Those numerical schemes are the MacCormack Scheme, the Fourth Order Runge Kutta Scheme, The First and Second Order Flux Splitting Steger Warming Scheme, Flux Splitting Bram Van Leer Scheme, the Harten Yee TVD scheme, Roe Sweby TVD scheme, Davies - Yee TVD scheme and the Modified Four Order Runge Kutta and Harten Yee TVD scheme. These nine numerical schemes are applied to the case of flow past through nozzle. The flow phenomena may appear along the nozzle may be the form completely as an isentropic flow or the flow with shock wave. These two flow phenomena are depended the boundary condition of the flow problem under considered. The implementation to case of isentropic flow along the nozzle, those nine numerical schemes works well, and the number of iterations required nearly the same between one with the other except for the Fourth Order Runge Kutta Scheme. In the case of flow with shock wave, the Fourth Order Runge Kutta Scheme, Second Order Steger Warming and the Bram Van Leer Scheme are failing. The presence of shock wave, each numerical scheme requires more iteration number to achieve a convergent solution. The Modified Runge Kutta - Harten Yee scheme may represent an appropriate a numerical scheme for solving the flow problem with the presence of shock wave.

Copyright $@ 2020$ PENERBIT AKADEMIA BARU - All rights reserved

Keywords:

Euler solver; TVD scheme; Steger warming scheme; Maccormack scheme

* Corresponding author.

E-mail address: nurhayati@uthm.edu.my (Nurhayati Rosly)

https://doi.org/10.37934/cfdl.12.6.93106 


\section{Introduction}

The governing equation of fluid motion modelled as inviscid flow model often used as the flow model for solving the aerodynamic problems in the aircraft design process. This is due to the fact that most aircraft configurations are designed to be a streamlined body immersed in the flow of high Reynolds number and low angle of attack. In such flow condition, the viscous effects can be ignored. The corresponding governing equation of fluid motion is known as the Euler Equations. The equation represents a system equation consist of the continuity equation, momentum and Energy equation. Basically, there are various numerical scheme had been developed for solving the Euler Equations. Hirsch [1] described the manner how to solve the Euler solver and can be grouped into two groups namely the Combined space - time discretization group of schemes and the Separate space discretization and time integration scheme. Each group have been developed into various numerical schemes. The Combined space - time integration had generated some numerical schemes such as numerical scheme based a space-centered spatial discretization scheme, or Upwind spatial discretization scheme or follow discretization according to the Total Variation Diminishing (TVD) criteria. While from the point of view time discretization or time integration, the numerical scheme may be in the form as explicit scheme, implicit scheme, single stage or a multistage time integration. Strictly speaking there are more than docent of Euler solvers had been developed and used for solving aerodynamics problems, however the present work just focusses on the following Euler Solvers, they are namely [1-4]
i. MacCormack scheme
ii. First order flux splitting Steger Warming scheme
iii. Second order flux splitting Steger Warming scheme
iv. First order flux splitting Bram Van Leer scheme
v. Fourth order Runge Kutta scheme
vi. Harten Yee TVD scheme
vii. Davies Yee TVD scheme
viii. Roe Sweby TVD scheme
ix. The modified Runge Kutta-Harten Yee TVD scheme

These nine numerical schemes are applied to two cases of flow past through divergent nozzle. The first case of divergent nozzle flow problem related to the problem of supersonic inflow supersonic outflow. So, the flow is flowing isentropic resulting the flow Mach number at the exit station is higher than at the entry station. The second flow problem relates to the problem of supersonic inflow and subsonic outflow. This flow condition describes the presence of shock wave somewhere inside the nozzle. Hence discontinuity flow phenomena appear in this flow problem.

The implementation of these nine numerical schemes to case of isentropic flow show all scheme work well. Comparison result in term of pressure and Mach number distribution along the nozzle are in good agreement each to others. The fourth order Runge Kutta scheme required a nearly 80000 iteration to achieve converge solution while other schemes just required the iteration process no more than 2000 iterations. In the case of flow with the presence of shock wave, three numerical schemes fail to converge. The Fourth Order Runge Kutta Scheme, Second Order Steger Warming and the Bram Van Leer Scheme represent the numerical scheme cannot be used when the flow field contains discontinuity flow phenomena. Strictly speaking, the Modified Runge Kutta - Harten Yee scheme may represent an appropriate a numerical scheme for solving the flow problem with the presence of shock wave compared with the other methods in the present study. 


\section{Methodology}

\subsection{Governing Equation of Fluid Motion along the Nozzle}

The governing equation of fluid motion along the nozzle's varying cross section $S(x)$ in assumption as unsteady inviscid one dimensional compressible can be written as $[2,5]$

$$
\frac{\partial S Q}{\partial t}+\frac{\partial F}{\partial x}-H=0
$$

Equation above is often called as the unsteady compressible one-dimensional Euler equation in vector notation. In that equation $Q$ is vector conserved variable, $F$ is the flux vector and $H$ is the vector of source term. These three vectors are

$Q=\left[\begin{array}{c}\rho \\ \rho u \\ \rho E\end{array}\right] ; F=S\left[\begin{array}{c}\rho u \\ \rho u+p \\ (\rho E+p) u\end{array}\right] ; H=\frac{d S}{d x}\left[\begin{array}{l}0 \\ p \\ 0\end{array}\right]$

The flow variables appears in above equations are the fluid density $\rho$, the flow velocity in $x$ direction is $u$, the pressure $p$ and the total internal energy $E$ represent the summation between internal energy $e$ and kinetic energy defined as

$E=e+\frac{1}{2} u^{2}$

Basically Eq. (2) describes a system equation which consist of three equation with four unknowns $(\rho, u, p$ and $E)$, hence additional one equation is needed to close that system equation. For a calorically perfect gas, there is a unique relation between pressure $\mathrm{p}$ and total internal energy $E$ as given by

$p=\rho(\gamma-1)\left(E-\frac{1}{2} u^{2}\right)$ or $E=\frac{p}{\rho(\gamma-1)}+\frac{1}{2} u^{2}$

And the relationship between fluid density $\rho$, pressure $p$ and temperature $T$ through equation of state defined as

$p=\rho R T$

In above, equation $\gamma$ is the ratio of heat coefficient and $\mathrm{R}$ is the universal gas constant.

\subsection{The Numerical Approach for Solving the Unsteady Compressible Euler Equations}

To solve the governing equation of fluid motion as given by Eq. (1), numerically by using finite difference method, a spatial discretization is required. The nozzle length starts from $x=0$ to $x=L$ need to be divided into $N$ number stations. In a simple way, the spatial discretization is carried out with the interval spacing is uniformly $\Delta x$. The numbering station start by number 1 at $x=1$ and end with the Nth station at $x=L$ as shown in the Figure 1 . 


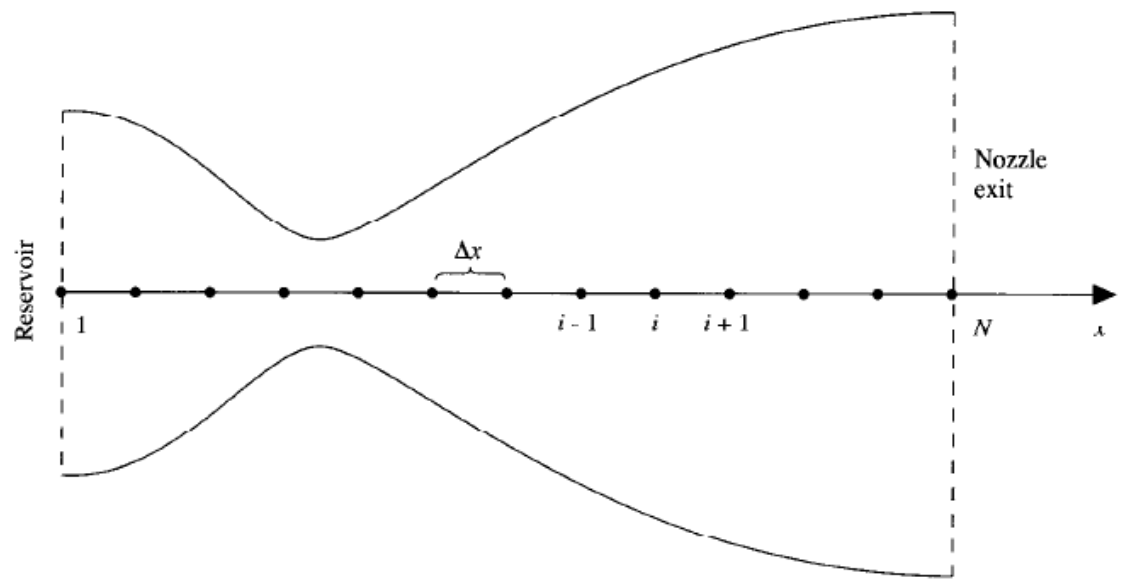

Fig.1. Spatial discretization along the nozzle

In solving the Euler equation, Eq. (1), the numerical schemes treat the flow problem as solving by use of a time marching process goes to the steady state solution. The solution start with a given initial condition at $t=0$ and the calculation proceed from one time step to other time and so if the difference result between two successive time steps $t=t_{n}$ and $t=t_{n}+\Delta t=t_{n+1}$ is less than a prescribed small number $\varepsilon$ the calculation is completed. Hoffmann [2] introduces that the convergent solution is completed if the total pressure difference between these two successive time steps fulfill the following relationship.

$\Delta p=\sum_{i=1}^{i=N}\left|p_{i}^{n+1}-p_{i}^{n}\right| \leq \varepsilon$

Strictly speaking, the mentioned numerical scheme as listed above had been discussed in various literatures, in this respect, one may refer to the Ref. 1 to Ref. 4 as result. Only some of them will be described shortly in the following subchapters.

\subsubsection{The MacCormack scheme $[3,5,6]$}

The MacCormack scheme represent a second-order finite difference method was introduced by Robert W. MacCormack in 1969 [6]. This scheme can be considered as variation of the two-step LaxWendroff scheme but is much simpler in application, elegant and easy to understand and as well as in converting into computer code. In manner how to solve the hyperbolic type of partial differential such as the governing equation of fluid motion along the nozzle as given by Eq. (1), the MacCormack scheme consist of two steps, the predictor step and the corrector step. The corrector step applied to the Eq. (1) becomes

Predictor step: $\frac{\partial S Q}{\partial t}+\frac{\partial F}{\partial x}-H=0 \quad \rightarrow\left(\frac{\partial Q}{\partial \bar{t}}\right)_{i}^{p}=-\frac{1}{S_{i}}\left[\frac{F_{i+1}^{n}-F_{i}^{n}}{\Delta x}\right]+\frac{1}{S_{i}} H_{i}^{n}$

Corrector step: $\frac{\partial S Q}{\partial t}+\frac{\partial F}{\partial x}-H=0 \rightarrow\left(\frac{\partial Q}{\partial \bar{t}}\right)_{i}^{c}=-\frac{1}{S_{i}}\left[\frac{F_{i}^{p}-F_{i-1}^{p}}{\Delta x}\right]+\frac{1}{s_{i}} H_{i}^{p}$

where $F_{i}^{p}$ and $F_{i}^{p}$ which they represent the flux vector and source term as defined by Eq. (2), their value are determined by the value of the conserved variable at the predictor step defined as 


$$
Q_{i}^{p}=Q_{i}^{p}+\left(\frac{\partial Q}{\partial \bar{t}}\right)_{i}^{p} \Delta t
$$

The conserved variables at the next following time step $t=t_{n+1}$ then can be obtained as

$$
Q_{i}^{n+1}=Q_{i}^{n}+\left(\frac{\partial Q}{\partial \bar{t}}\right)_{i}^{a v e} \Delta t
$$

where

$$
\left(\frac{\partial \boldsymbol{Q}}{\partial \bar{t}}\right)_{i}^{a v e}=0.5\left[\left(\frac{\partial \boldsymbol{Q}}{\partial \bar{t}}\right)_{i}^{p}+\left(\frac{\partial \boldsymbol{Q}}{\partial \bar{t}}\right)_{i}^{c}\right]
$$

\subsubsection{The fourth order Runge Kutta scheme $[2,6]$}

The Runge-Kutta scheme is similar to the MacCormack schemes, this scheme can be considered as well as belong to the class of predictor-corrector methods. However, this scheme may represent is the most powerful predictor-correctors methods since this approach can be developed as a single predictor step followed by one or more corrector steps. A second order Runge Kutta scheme is the scheme with a single predictor step then followed by one corrector step. While third order Runge Kutta Scheme consist of a single predictor with two corrector step and the Fourth order as the method with a single predictor then followed by three corrector steps. The implementation Fourth order Runge Kutta Scheme into the governing equation of fluid motion along the nozzle makes Eq. (1) can be written as

$$
\begin{aligned}
& Q_{i}^{(1)}=Q_{i}^{n} \\
& Q_{i}^{(2)}=Q_{i}^{n}-\frac{\Delta t}{4}\left(\frac{1}{s_{i}}\right)\left[\left(\frac{\partial F}{\partial x}\right)_{i}^{(1)}-H_{i}^{(1)}\right] \\
& Q_{i}^{(3)}=Q_{i}^{n}-\frac{\Delta t}{4}\left(\frac{1}{s_{i}}\right)\left[\left(\frac{\partial F}{\partial x}\right)_{i}^{(2)}-H_{i}^{(2)}\right] \\
& Q_{i}^{(4)}=Q_{i}^{n}-\frac{\Delta t}{4}\left(\frac{1}{s_{i}}\right)\left[\left(\frac{\partial F}{\partial x}\right)_{i}^{(3)}-H_{i}^{(3)}\right] \\
& Q_{i}^{n+1}=Q_{i}^{n}-\frac{\Delta t}{4}\left(\frac{1}{s_{i}}\right)\left[\left(\frac{\partial F}{\partial x}\right)_{i}^{(4)}-H_{i}^{(4)}\right]
\end{aligned}
$$

In above equation the spatial derivative $\left(\frac{\partial F}{\partial x}\right)_{i}^{(n)}, n=1,2,3,4$ is approximated by use of second order central difference approximation, namely

$$
\left(\frac{\partial F}{\partial x}\right)_{i}^{(n)}=\frac{F_{i+1}^{(n)}-F_{i-1}^{(n)}}{2 \Delta x}
$$




\subsubsection{First and second order upwind schemes [2,7]}

The Steger Warming and Bram Van Leer scheme, they are basically the scheme belong to the class upwind scheme if one look them in the point of view their spatial discretization in used. To understand these methods, let consider a simple model of hyperbolic partial differential equation called wave equation. This equation is defined as

$\frac{\partial u}{\partial t}+c \frac{\partial u}{\partial x}=0$

where $c$ is constant value and the initial condition at time $t=0$ is

$u(x, 0)=u_{0}(x)$

The analytic solution of hyperbolic partial differential as above is

$u(x, t)=u_{0}(x-c t)$

The solution as described by Eq. (14) explains that the solution at any time $t$ is a copy of the original function, but shifted to the right, if $c$ is positive, or to the left, if $c$ is negative, by an amount $|c| t$. In other word if the constant $c$ is positive, the dependent variable $u$ at the right position is depended on the information come from its left side while if $c$ negatives, the dependent variable $u$ in the left come from the right side. As result a Forward time backward space discretization approach which often called as upwind scheme can be implemented namely

First order Upwind scheme

$$
\begin{aligned}
& \frac{\partial u}{\partial t}+c \frac{\partial u}{\partial x}=0 \rightarrow \frac{u_{i}^{n+1}-u_{i}^{n}}{\Delta t}=-c \frac{u_{i}^{n}-u_{i-1}^{n}}{\Delta x} \text { for } c>0 \text { or } \\
& \frac{u_{i}^{n+1}-u_{i}^{n}}{\Delta t}=-c \frac{u_{i+1}^{n}-u_{i}^{n}}{\Delta x} \text { for } c<0
\end{aligned}
$$

Second order Upwind scheme,

$$
\begin{aligned}
& \frac{\partial u}{\partial t}+c \frac{\partial u}{\partial x}=0 \rightarrow \frac{u_{i}^{n+1}-u_{i}^{n}}{\Delta t}=-c \frac{3 u_{i}^{n}-4 u_{i-1}^{n}+u_{i-2}^{n}}{2 \Delta x} \text { for } c>0 \text { or } \\
& \frac{u_{i}^{n+1}-u_{i}^{n}}{\Delta t}=-c \frac{-u_{i+2}^{n}-4 u_{i+1}^{n}+3 u_{i}^{n}}{2 \Delta x} \text { for } c<0
\end{aligned}
$$

Basically Steger Warming scheme [7] try to apply the Upwind scheme in solving the Euler equation through splitting flux terms to becomes the flux vector $\mathrm{F}$ can be written as $\mathrm{F}^{+}$and $\mathrm{F}^{-}$and so the discrete form of the Euler equation, Eq. (1) in point of view as the first order upwind scheme as

$$
\begin{aligned}
& \frac{\partial S Q}{\partial t}+\frac{\partial F}{\partial x}-H=0 \rightarrow \quad Q_{i}^{n+1}=Q_{i}^{n}-\left(\frac{1}{s_{i}}\right)\left(\frac{\Delta t}{\Delta x}\right)\left[\left(F^{+}\right)_{i}^{n}-\left(F^{+}\right)_{i}^{n}+\left(F^{+}\right)_{i}^{n}-\left(F^{+}\right)_{i}^{n}\right]+ \\
& \left(\frac{1}{s_{i}}\right) \Delta t H_{i}^{n}
\end{aligned}
$$


while as the second order upwind scheme becomes

$Q_{i}^{n+1}=Q_{i}^{n}-\left(\frac{1}{s_{i}}\right)\left(\frac{\Delta t}{2 \Delta x}\right)\left[\left(E^{+}\right)_{i-2}^{n}-4\left(F^{+}\right)_{i}^{n}+3\left(F^{+}\right)_{i}^{n}-3\left(E^{-}\right)_{i}^{n}+4\left(F^{-}\right)_{i}^{n}-3\left(F^{-}\right)_{i+2}^{n}\right]+$
$\left(\frac{1}{s_{i}}\right) \Delta t H_{i}^{n}$

The Steger Warming in determining the positive flux vector $F^{+}$and $F^{-}$are based on the eigenvalues of the Jacobian matrix $[A]=\left[\frac{\partial F}{\partial Q}\right]$. For the case of one dimensional flow such as the flow past through nozzle, the eigenvalue of that matrix is $[u-a, u, u+a]]$, where $u$ is the component velocity in $\mathrm{x}$-direction and $a$ is the local speed of sound. Hence for a supersonic flow these three eigenvalues are positive and for the subsonic flow one of its eigenvalue $(u-a)$ is negative. Based on the value of its eigenvalue, Steger-Warming for the supersonic flow condition (Mach number $M>1$ ), the positive flux vector $F^{+}$and the negative flux vector $F^{-}$becomes

$F^{+}=F=S\left[\begin{array}{c}\rho u \\ \rho u+p \\ (\rho E+p) u\end{array}\right]$ and $F^{-}=\left[\begin{array}{l}0 \\ 0 \\ 0\end{array}\right]$ for $M>0$

while for subsonic flow condition $M<1$, those two flux vectors are

$F^{+}=S \frac{\rho}{2 \gamma}\left[\begin{array}{c}2 \gamma+a-u \\ 2(\gamma-1) u^{2}+(u+a)^{2} \\ (\gamma-1) u^{3}+\frac{1}{2}(u+a)^{3}+\left[(3-\gamma)(u+a) a^{2}\right] /[2(\gamma-1)]\end{array}\right]$

and

$F^{-}=S \frac{\rho}{2 \gamma}\left[\begin{array}{c}u-a \\ (u-a)^{2} \\ \frac{1}{2}(u-a)^{3}+\left[(3-\gamma)(u-a) a^{2}\right] /[2(\gamma-1)]\end{array}\right]$

while Bram Van Leer [2,8] defines the splitted flux vector $F$ into $F^{+}$and $F^{-}$are respectively as [3]

$F^{+}=S \frac{1}{4} \rho a(1+M)^{2}\left[\begin{array}{c}a\left(\frac{\gamma-1}{\gamma} M+\frac{2}{\gamma}\right) \\ \frac{1}{2} a^{2} \frac{\gamma^{2}}{\gamma^{2}-1}\left(\frac{\gamma-1}{\gamma} M+\frac{2}{\gamma}\right)^{2}\end{array}\right] \quad$ for $-1<M<1$

and

$F^{-}=-S \frac{1}{4} \rho a(1-M)^{2}\left[\begin{array}{c}1 \\ -a\left(-\frac{\gamma-1}{\gamma} M+\frac{2}{\gamma}\right) \\ -\frac{1}{2} a^{2} \frac{\gamma^{2}}{\gamma^{2}-1}\left(-\frac{\gamma-1}{\gamma} M+\frac{2}{\gamma}\right)^{2}\end{array}\right] \quad$ for $-1<M<1$

while for $M \leq-1$ and $M \geq 1$ those two flux vectors become 
$F^{+}=0$ and $F^{-}=F=S\left[\begin{array}{c}\rho u \\ \rho u+p \\ (\rho E+p) u\end{array}\right] \quad$ for $M \leq-1$

and

$F^{+}=F=S\left[\begin{array}{c}\rho u \\ \rho u+p \\ (\rho E+p) u\end{array}\right]$ and $F^{-}=0 \quad$ for $M \geq 1$

Detail how to derive $F^{ \pm}$may one refers to Van Leer [6].

\subsubsection{Total variation diminishing scheme $[2,9,10]$}

The Harten-Yee $[2,9,11]$, Davies -Yee $[2,12]$ and Roe-Sweby $[2,13,14]$ scheme has the same starting point in solving the Euler equations. They assume that if the total variation $T V\left(u^{n}\right)$ defined as [7]

$T V\left(u^{n}\right)=\sum_{i=1}^{i=I \max }\left\lceil u_{i+1}^{n}-u_{i}^{n}\right\rceil$

with the total variation between the solution between two successive time steps fulfil the following condition

$T V\left(u^{n+1}\right) \leq T V\left(u^{n}\right)$

This condition one can arrive that the Euler equation as given by Eq. (1) can be discretised in form

$Q_{i}^{n+1}=Q_{i}^{n}-\frac{1}{s_{i}}\left(\frac{\Delta t}{\Delta x}\right)\left[R_{i+\frac{1}{2}}^{n}-R_{i+\frac{1}{2}}^{n}\right\rceil+\frac{\Delta t}{s_{i}} H_{i}^{n}$

where

$R_{i+\frac{1}{2}}^{n}=\frac{1}{2}\left\lceil F_{i+1}^{n}+F_{i}^{n}+X_{i+\frac{1}{2}}^{n} \Phi_{i+\frac{1}{2}}^{n}\right\rceil$

and

$R_{i-\frac{1}{2}}^{n}=\frac{1}{2}\left\lceil F_{i+1}^{n}+F_{i}^{n}+X_{i-\frac{1}{2}}^{n} \Phi_{i-\frac{1}{2}}^{n}\right\rceil$

Two additional terms $X$ and $\Phi$ in above equation represents the eigenvector and the flux limiter vector. The eigenvector matrix for the Jacobian matrix $A=\left[\frac{\partial F}{\partial Q}\right]$ is defined as

$X=\left[\begin{array}{ccc}1 & \alpha & \alpha \\ u & \alpha(u+a) & \alpha(u-a) \\ \frac{1}{2} u^{2} & \alpha\left(\frac{1}{2} u^{2}+u a+\frac{a^{2}}{\gamma-1}\right) & \alpha\left(\frac{1}{2} u^{2}-u a-\frac{a^{2}}{\gamma-1}\right)\end{array}\right]$

where 
$\alpha=\frac{\rho}{a \sqrt{2}}$

The flux limiter vector $\Phi$ which makes differences between Harten-Yee TVD scheme with the Davies - Yee TVD scheme as well as with Roe -Sweby TVD scheme. Davis-Yee's flux limiter vector is given below while the other two flux limiter may one can refer to the Hoffman CFD textbook [3].

$$
\Phi_{i+\frac{1}{2}}^{n}=-\frac{1}{2}\left\lceil\left(\frac{\Delta t}{\Delta x}\right)\left(\alpha_{i+\frac{1}{2}}^{n}\right)^{2} G_{i+\frac{1}{2}}^{n}+\Psi\left(\alpha_{i+\frac{1}{2}}^{n}\right)\left(\delta_{i+\frac{1}{2}}^{n}-G_{i+\frac{1}{2}}^{n}\right)\right\rceil
$$

and

$$
\Phi_{i-\frac{1}{2}}^{n}=-\frac{1}{2}\left\lceil\left(\frac{\Delta t}{\Delta x}\right)\left(\alpha_{i-\frac{1}{2}}^{n}\right)^{2} G_{i-\frac{1}{2}}^{n}+\Psi\left(\alpha_{i-\frac{1}{2}}^{n}\right)\left(\delta_{i-\frac{1}{2}}^{n}-G_{i-\frac{1}{2}}^{n}\right)\right\rceil
$$

The limiter $G_{i+\frac{1}{2}}^{n}$ may one can choose one of three following models

$$
\begin{aligned}
& G_{i+\frac{1}{2}}^{n}=\operatorname{minmod}\left[2 \delta_{i-\frac{1}{2}}^{n}, 2 \delta_{i+\frac{1}{2}}^{n}, 2 \delta_{i+\frac{3}{2}}^{n}, \frac{1}{2}\left(\delta_{i-\frac{1}{2}}^{n}+\delta_{i+\frac{3}{2}}^{n}\right)\right] \\
& G_{i+\frac{1}{2}}^{n}=\operatorname{minmod}\left[\delta_{i-\frac{1}{2}}^{n}, \delta_{i+\frac{1}{2}}^{n}, 2 \delta_{i+\frac{3}{2}}^{n}\right] \\
& G_{i+\frac{1}{2}}^{n}=\operatorname{minmod}\left[\delta_{i+\frac{1}{2}}^{n}, \delta_{i-\frac{1}{2}}^{n}\right]+\operatorname{minmod}\left[\delta_{i+\frac{1}{2}}^{n}, \delta_{i+\frac{3}{2}}^{n}\right]-\delta_{i+\frac{1}{2}}^{n}
\end{aligned}
$$

$\delta_{i+\frac{1}{2}}^{n}=\left[X^{-1}\right]\left[Q_{i+1}^{n}-Q_{i}^{n}\right]$

$\left[X^{-1}\right]=\left[\begin{array}{ccc}1-\frac{1}{2} u^{2} \frac{\gamma-1}{a^{2}} & (\gamma-1) \frac{u^{2}}{a^{2}} & -\frac{(\gamma-1)}{a^{2}} \\ \beta\left((\gamma-1) \frac{u^{2}}{2}-u a\right) & \beta(\alpha-(\gamma-1) u) & \beta(\gamma-1) \\ \beta\left((\gamma-1) \frac{u^{2}}{2}+u a\right) & -\beta(\alpha-(\gamma-1) u) & \beta(\gamma-1)\end{array}\right]$

$\beta=1 /(\rho a \sqrt{2})$

\subsubsection{The modified four order Runge Kutta - Harten Yee TVD [2,9]}

This scheme represents the combination of Fourth Order Runge Kutta Scheme and Harten Yee TVD scheme. The calculation process from one time step to next following time step namely from $t=t_{n}$ to $t=t_{n+1}$ firstly carried out by use the Fourth Order Runge Kutta Scheme however their result still considered as the result at time level $t=t_{n}$. This value then will be used by Harten Yee scheme to define the flow variables for the time level $=t_{n+1}$. 


\section{Discussion and Results}

To evaluate those Euler solvers as mentioned, the present work uses a nozzle model as introduced by Hoffmann [2] other nozzle model may one refer to the work of Nasir [15]. This model is a divergent nozzle defined to have a circular cross section with cross section area distribution along the nozzle $x$-axis is given as

$S(x)=1.398+0.347 \tanh (0 . x-4.0)$ for $0 \leq x \leq 10$

The flow problems may appear in the form

i. Supersonic inflow - supersonic out flow

ii. Supersonic inflow - subsonic outflow

The first case, Supersonic inflow - supersonic outflow, means the flow expands along the nozzle in isentropic manner and the flow Mach number at the exit station is higher than at the entry station. While the second case deals with the presence of shock wave somewhere appear inside the nozzle which make the flow speed at the exit station is a subsonic flow. The following subchapter discuss the implementation of the various scheme as mentioned above in solving these two nozzle flow problems.

\subsection{Case of Nozzle's Flow Problems: Supersonic Inflow - Supersonic Outflow}

In supersonic inflow - supersonic outflow for a given nozzle geometry one can define their distribution of Mach number, pressure, density as well as their distribution of the temperature along the $x$-axis of the nozzle. For a given cross section area $S(x)$, Mach number $M$ at any position $x$ can be obtained through solving the following relations.

$\frac{S(x)}{S^{*}}=\frac{1}{M}\left[\frac{2}{\gamma+1}\left(1+\frac{\gamma-1}{2} M^{2}\right)\right]^{0.5\left(\frac{\gamma+1}{\gamma-1}\right)}$

For known Mach number and the flow is isentropic, so the flow variables can be determined as

$$
\begin{aligned}
& T=T_{01}\left(1+\frac{\gamma-1}{2} M^{2}\right)^{-1} \\
& p=p_{01}\left(1+\frac{\gamma-1}{2} M^{2}\right)^{-\frac{\gamma}{\gamma-1}} \\
& \rho=\rho_{01}\left(1+\frac{\gamma-1}{2} M^{2}\right)^{-\frac{1}{\gamma-1}}
\end{aligned}
$$

The subscribe 01 is used to describe that the corresponding the flow quantity at its stagnation flow condition. $S^{*}$ is the cross-section area in which at that the cross section the flow is reached a chocked flow condition. As the case of supersonic inflow and supersonic outflow, at the entry station, the flow variables must be define and at exit station no prescribed value of flow variables are needed.

The flow condition at the entry station $x=0$ is 
The flow Mach number $M_{1}=1.5$

The static pressure $p_{1}=2000 \frac{l b f}{f t^{2}}$

The static temperature $T=520^{\circ}$ Reamur

At the entry station $x_{1}=0$, the cross section area $S_{1}$ is equal to $1.051 \mathrm{ft}^{2}$. For a given $S_{1}$ and $M_{1}$, using Eq. (32) one can determine $S^{*}$. As the $S^{*}$ is known, the ratio of cross section area at any station $S(x)$ to the $S^{*}$ can be defined and by using equation (32) again can be used to determine the value of Mach number M. Knowing the Mach number $M$ then by using Eq. (33), one can define the pressure $p$ and temperature T. The fluid density $\rho$ for known $p$ and $T$, then can be obtained from the equation of state namely

$\rho=\frac{p}{R T}$

where $\mathrm{R}$ is universal gas constant. It is equal to $1716 \frac{\mathrm{lbf} . f t}{\operatorname{slug}{ }^{0} R}$ in the British unit.

The comparison results between numerical schemes as mentioned above in term of Mach number distribution along the $\mathrm{x}$-axis of the nozzle as well as in term of pressure and density are shown in the Figure 2(a) -2 (d) respectively. Considering these three figures it is clear that all mentioned numerical scheme have capability in solving the Euler equation.

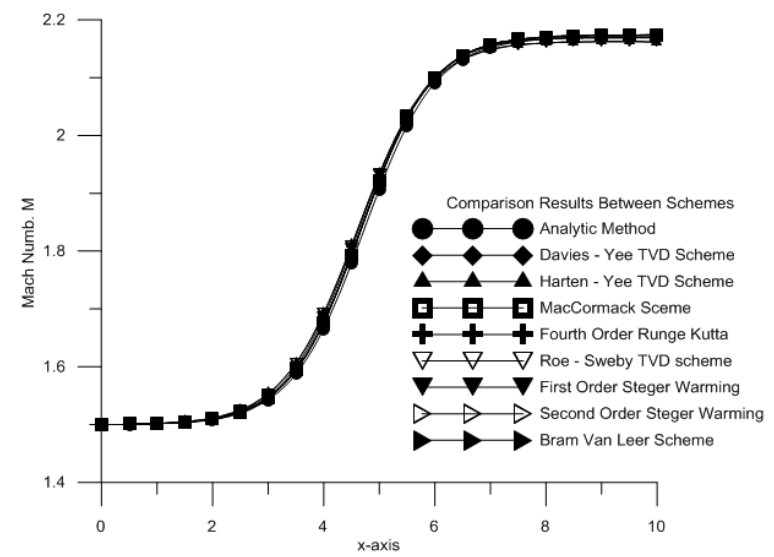

(a)

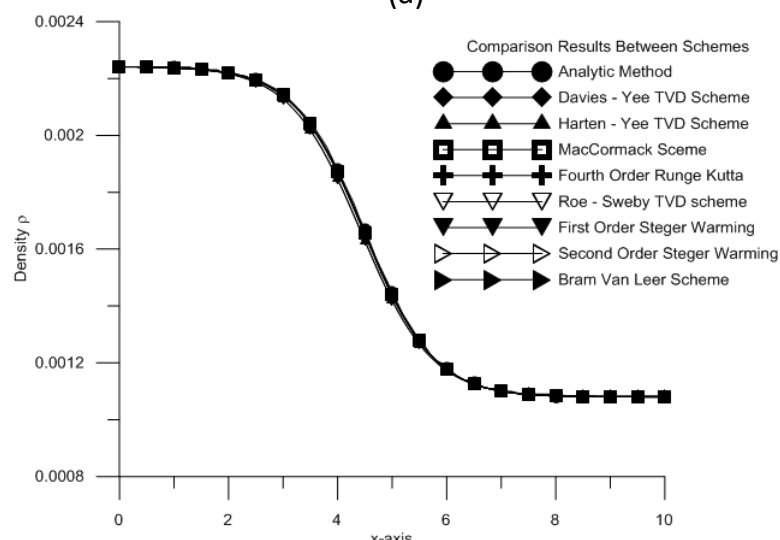

(c)

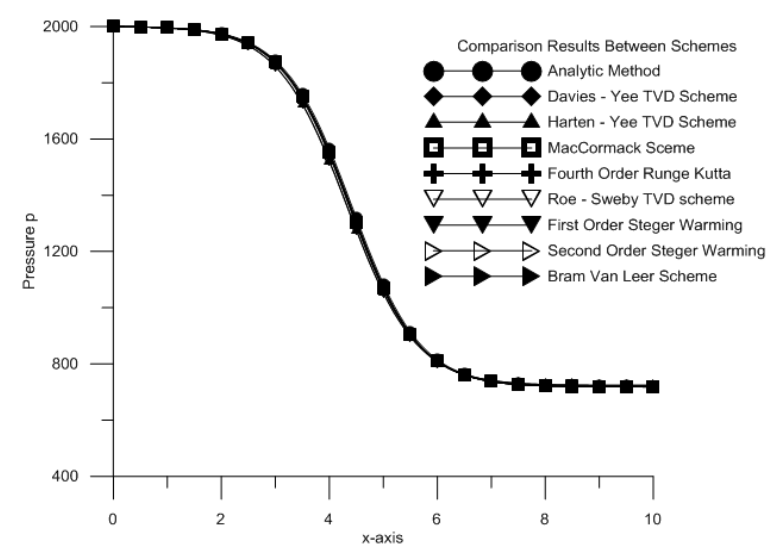

(b)

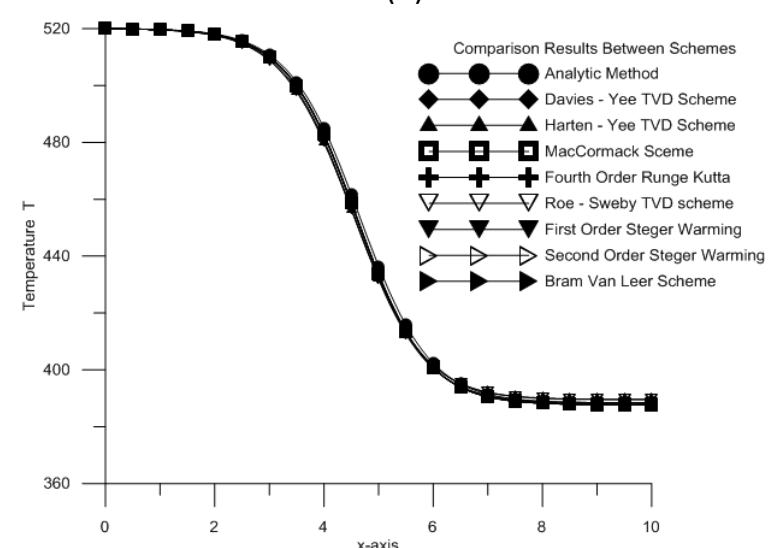

(d)

Fig. 2. The (a) Mach number distribution, (b) pressure distribution, (c) density $\rho$ distribution (c) and (d) temperature distribution along the $x$-axis nozzle 
It is necessary to be noted, the numerical parameter had been applied in carrying out calculations, namely the setting spatial discretization use a uniform spacing with total number of control point distributed along $\mathrm{x}$-axis is equal to 101 points. The uniform time step $\Delta \mathrm{t}$ is set equal to 0.00001 unit time. The convergence criteria which is defined by Eq. (6) by setting the value of $\varepsilon=0.01$. Such numerical parameter makes the required iteration number to converge between one scheme with other scheme as shown in the Table 1.

\section{Table 1}

The required number of iterations for converge solution

\begin{tabular}{lll}
\hline No & Numerical Scheme & Iteration number \\
\hline 1 & Davies - Yee TVD Scheme & 1090 \\
2 & Harten - Yee TVD Scheme & 1167 \\
3 & MacCormack Scheme & 1667 \\
4 & Fourth Order Runge Kutta Scheme & 80001 \\
5 & Roe - Sweby TVD Scheme & 1162 \\
6 & First Order Steger Warming Scheme & 1126 \\
7 & Second Order Steger Warming & 1047 \\
8 & Bram Van Leer Scheme & 1077 \\
\hline
\end{tabular}

\subsection{Case of Nozzle's flow Problems: Supersonic Inflow - Subsonic Outflow}

In the case of the presence of shock wave, the back pressure $P_{b}$ which represent the pressure at the exist station must be greater than certain value, namely for a given the entry condition as given in the previous sub chapter, the back pressure must be greater than .720 $\frac{l b f}{f t^{2}}$. The following test use the same flow condition at the entry station, but for the exit station by setting that the flow speed at the exit station is equal to $572.76 \mathrm{ft} / \mathrm{sec}$. By using a similar a numerical parameter as in the previous subchapter, it had been found that some numerical scheme diverges and some of them are successfully converge as shown in the Table 2.

Table 2

The required number of iterations for converge solution

\begin{tabular}{lll}
\hline No & Numerical Scheme & Iteration number \\
\hline 1 & Davies - Yee TVD Scheme & 9669 \\
2 & Harten - Yee TVD Scheme & 9117 \\
3 & MacCormack Scheme & 6553 \\
4 & Fourth Order Runge Kutta Scheme & fail \\
5 & Roe - Sweby TVD Scheme & 9026 \\
6 & First Order Steger Warming Scheme & 8491 \\
7 & Second Order Steger Warming & fail \\
8 & Bram Van Leer Scheme & fail \\
9 & Modified Runge Kutta + Harten Yee & 5078 \\
\hline
\end{tabular}

The comparison results between the numerical scheme which successfully goes to converge solution in term of Mach number and pressure distribution as shown in the Figure 3a and Figure $3 \mathrm{~b}$. Considering figures below, it is clear that those six numerical schemes have a good capability in capturing the presence of shock wave in the flow field. 


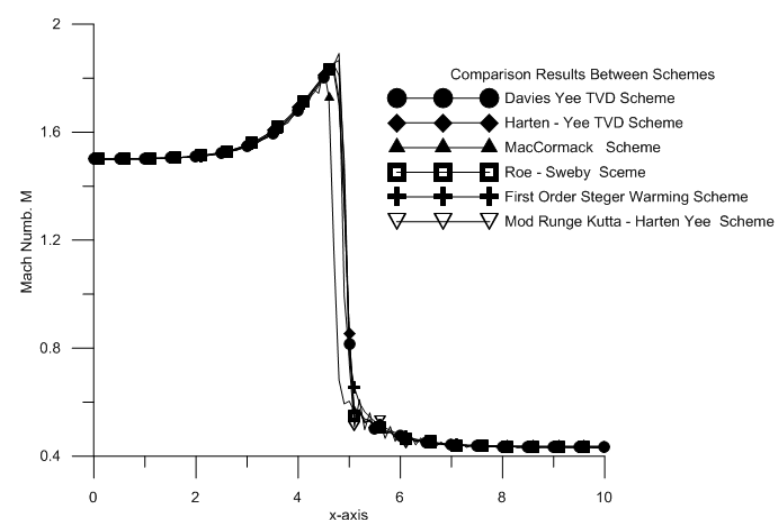

(a)

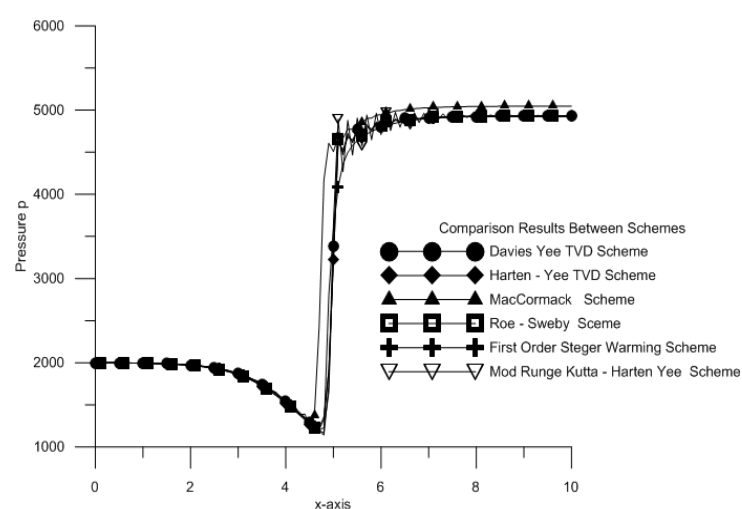

(b)

Fig. 3. The (a) Mach number Distribution and (b) Pressure Distribution along the $x$-axis nozzle

\section{Conclusions}

Considering the result as shown in sub chapter 4.1 is clear that the all numerical schemes as mentioned above work well for smooth flow problem. The Second Order Runge Kutta Scheme offer the lowest number of required iteration process for achieving a convergence solution. It is necessary to be noted, the Fourth order Runge Kutta scheme is actually failed, if the convergence criteria is set below 0.01 . In the case of non-smooth flow problems or the flow with the presence of shock wave in the flow field, it can be said that all numerical scheme which belong to the class of TVD scheme are converged but most of them require a high iteration number. The Modified Runge Kutta - Harten Yee scheme may represent an appropriate a numerical scheme for solving the flow problem with the presence of shock wave compared with the other methods in the present study.

\section{Acknowledgement}

The present author acknowledges to the Universiti Tun Hussain Onn (UTHM) in providing the financial support through TIER-1 Grant H140 which allowing this research work can be conducted.

\section{References}

[1] Hirsch, Charles. "Numerical computation of internal and external flows, Volume 1: Fundamentals of numerical discretization." John Wiley and Sons 9 (1988): 10.

[2] Hoffmann, Klaus A., and Steve T. Chiang. "Computational fluid dynamics volume I." Engineering Education System (2000).

[3] Anderson, John David, and J. Wendt. Computational fluid dynamics. Vol. 206. New York: McGraw-Hill, 1995.

[4] Fletcher, Clive AJ. "Fluid Dynamics: The Governing Equations." In Computational Techniques for Fluid Dynamics 2 , pp. 1-46. Springer, Berlin, Heidelberg, 1991.

https://doi.org/10.1007/978-3-642-58239-4 1

[5] Ferziger, Joel H., Milovan Perić, and Robert L. Street. Computational methods for fluid dynamics. Vol. 3. Berlin: springer, 2002.

https://doi.org/10.1007/978-3-642-56026-2

[6] Pletcher, Richard H., John C. Tannehill, and Dale Anderson. Computational fluid mechanics and heat transfer. CRC press, 2012.

[7] Steger, Joseph L., and R. F. Warming. "Flux vector splitting of the inviscid gasdynamic equations with application to finite-difference methods." Journal of computational physics 40, no. 2 (1981): 263-293. https://doi.org/10.1016/0021-9991(81)90210-2

[8] Van Leer, B. "Flux vector splitting for the 1990's." In Proceedings of the Computational Fluid Dynamics Symposium on Aeropropulsion, pp. 203-214. NASA CP-3078, Cleveland, OH, 1990.

[9] Yee, Helen C. "Explicit and implicit multidimensional compact high-resolution shock-capturing methods: formulation." Journal of Computational Physics 131, no. 1 (1997): 216-232.

https://doi.org/10.1006/jcph.1996.5608 
[10] Harten, Amiram. "The artificial compression method for computation of shocks and contact discontinuities. III. Selfadjusting hybrid schemes." Mathematics of Computation 32, no. 142 (1978): 363-389.

https://doi.org/10.2307/2006149

[11] Yee, Helen C. "Upwind and symmetric shock-capturing schemes." (1987).

[12] Davis, Stephen F. "TVD finite difference schemes and artificial viscosity." (1984).

[13] Sweby, Peter K. "High resolution schemes using flux limiters for hyperbolic conservation laws." SIAM journal on numerical analysis 21, no. 5 (1984): 995-1011. https://doi.org/10.1137/0721062

[14] Roe, P. L. "Generalized formulation of TVD Lax-Wendroff schemes." (1984).

[15] Hassen, Nasir Salim, and Nor Azwadi Che Sidik. "Laboratory Investigation of Nozzle Type, Size and Pressure Effects on Spray Distribution." Journal of Advanced Research in Fluid Mechanics and Thermal Sciences 61, no. 1 (2019): 140-146. 